Draft Version MARch 5, 2021

Typeset using $\mathrm{LAT}_{\mathrm{E}} \mathrm{X}$ twocolumn style in AASTeX63

\title{
First Detection of Hydroxyl Radical Emission from an Exoplanet Atmosphere: High-dispersion Characterization of WASP-33b using Subaru/IRD*
}

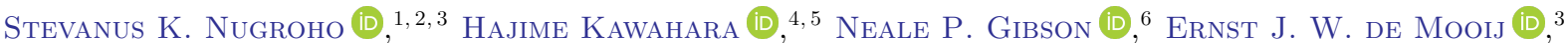 \\ Teruyuki Hirano (D), ${ }^{1,2}$ Takayuki Kotani (D), ${ }^{1,2,7}$ Yui Kawashima $\mathbb{D}$, ${ }^{8}$ Kento Masuda (D), ${ }^{9}$ Matteo Brogi (D), ${ }^{10,11,12}$ \\ Jayne L. Birkby (D), ${ }^{13}$ Chris A. Watson $\mathbb{D},{ }^{3}$ Motohide Tamura (D), ${ }^{14,1,2}$ Konstanze Zwintz (D), ${ }^{15}$ \\ Hiroki Harakawa (D) ${ }^{16}$ Tomoyuki Kudo $\left(\mathbb{D},{ }^{16}\right.$ Masayuki Kuzuhara $\left(\mathbb{D},{ }^{1,2}\right.$ Klaus Hodapp, ${ }^{17}$ Masato Ishizuka (D), ${ }^{14}$ \\ Shane Jacobson, ${ }^{17}$ Minoko Konishi, ${ }^{18}$ Takashi Kurokawa, ${ }^{1,19}$ Jun Nishikawa $\mathbb{D}^{2,}, 1,7$ Masashi Omiya (D), ${ }^{1,2}$ \\ Takuma Serizawa, ${ }^{19,2}$ Akitoshi Ueda,,${ }^{1,2,7}$ and Sébastien Vievard (id ${ }^{1,16}$ \\ ${ }^{1}$ Astrobiology Center, NINS, 2-21-1 Osawa, Mitaka, Tokyo 181-8588, Japan \\ ${ }^{2}$ National Astronomical Observatory of Japan, NINS, 2-21-1 Osawa, Mitaka, Tokyo 181-8588, Japan \\ ${ }^{3}$ School of Mathematics and Physics, Queen's University Belfast, University Road, Belfast, BT7 1NN, United Kingdom \\ ${ }^{4}$ Department of Earth and Planetary Science, The University of Tokyo, Tokyo 113-0033, Japan \\ ${ }^{5}$ Research Center for the Early Universe, School of Science, The University of Tokyo, Tokyo 113-0033, Japan \\ ${ }^{6}$ School of Physics, Trinity College Dublin, The University of Dublin, Dublin 2, Ireland \\ ${ }^{7}$ Department of Astronomy, School of Science, The Graduate University for Advanced Studies (SOKENDAI), 2-21-1 Osawa, Mitaka, \\ Tokyo 181-8588, Japan \\ ${ }^{8}$ Cluster for Pioneering Research, RIKEN, 2-1 Hirosawa, Wako, Saitama 351-0198, Japan \\ ${ }^{9}$ Department of Earth and Space Science, Osaka University, Osaka 560-0043, Japan \\ ${ }^{10}$ Department of Physics, University of Warwick, Coventry CV4 7AL, UK \\ ${ }^{11}$ INAF-Osservatorio Astrofisico di Torino, Via Osservatorio 20, I-10025, Pino Torinese, Italy \\ ${ }^{12}$ Centre for Exoplanets and Habitability, University of Warwick, Gibbet Hill Road, Coventry CV4 7AL, UK \\ ${ }^{13}$ Astrophysics, Department of Physics, University of Oxford, Keble Road, Oxford OX1 3RH, UK \\ ${ }^{14}$ Department of Astronomy, Graduate School of Science, The University of Tokyo, 7-3-1 Hongo, Bunkyo-ku, Tokyo 113-0033, Japan \\ ${ }^{15}$ Institute for Astro- and Particle Physics, University of Innsbruck, Technikerstrasse 25/8, A-6020 Innsbruck, Austria \\ ${ }^{16}$ Subaru Telescope, 650 N. Aohoku Place, Hilo, HI 96720, USA \\ ${ }^{17}$ University of Hawaii, Institute for Astronomy, 640 N. Aohoku Place, Hilo, HI 96720, USA \\ ${ }^{18}$ Faculty of Science and Technology, Oita University, 700 Dannoharu, Oita 870-1192, Japan \\ ${ }^{19}$ Institute of Engineering, Tokyo University of Agriculture and Technology, 2-24-16, Nakacho, Koganei, Tokyo, 184-8588, Japan
}

(Received February 12, 2021; Revised March 3, 2021; Accepted March 4, 2021)

Submitted to ApJL

\begin{abstract}
We report the first detection of a hydroxyl radical $(\mathrm{OH})$ emission signature in the planetary atmosphere outside the solar system, in this case, in the day-side of WASP-33b. We analyze high-resolution near-infrared emission spectra of WASP-33b taken using the InfraRed Doppler spectrograph on the 8.2-m Subaru telescope. The telluric and stellar lines are removed using a de-trending algorithm, SysRem. The residuals are then cross-correlated with $\mathrm{OH}$ and $\mathrm{H}_{2} \mathrm{O}$ planetary spectrum templates produced using several different line-lists. We check and confirm the accuracy of $\mathrm{OH}$ line-lists by cross-correlating with the spectrum of GJ 436. As a result, we detect the emission signature of $\mathrm{OH}$ at $K_{\mathrm{p}}$ of $230.9_{-7.4}^{+6.9} \mathrm{~km} \mathrm{~s}^{-1}$ and $v_{\mathrm{sys}}$ of $-0.3_{-5.6}^{+5.3} \mathrm{~km} \mathrm{~s}^{-1}$ with $\mathrm{S} / \mathrm{N}$ of 5.4 and significance of $5.5 \sigma$. Additionally, we marginally detect $\mathrm{H}_{2} \mathrm{O}$ emission in the $\mathrm{H}$-band with $\mathrm{S} / \mathrm{N}$ of 4.0 and significance of $5.2 \sigma$ using the POKAZATEL line-list. However, no significant signal is detected using the HITEMP 2010, which might be due to differences in line positions and strengths, as well as the incompleteness of the
\end{abstract}

Corresponding author: Stevanus K. Nugroho

stevanus.nugroho@nao.ac.jp, skristiantonugroho@gmail.com

* Based on data collected at Subaru Telescope, which is operated by the National Astronomical Observatory of Japan. 
line-lists. Nonetheless, this marginal detection is consistent with the prediction that $\mathrm{H}_{2} \mathrm{O}$ is mostly thermally dissociated in the upper atmosphere of the ultra-hot Jupiters. Therefore, along with CO, $\mathrm{OH}$ is expected to be one of the most abundant O-bearing molecules in the day-side atmosphere of ultra-hot Jupiters and should be considered when studying their atmosphere.

Keywords: Exoplanet atmospheres (487); Exoplanet atmospheric composition (2021); High resolution spectroscopy (2096)

\section{INTRODUCTION}

High-resolution spectroscopy is one of the most successful methods to characterize exoplanet atmospheres, especially of hot Jupiters. The resolved planetary lines are disentangled from the telluric and stellar lines due to the planetary orbital motion allowing us to unambiguously detect atomic/molecular signatures in the atmosphere of exoplanets (e.g., Snellen et al. 2010). By comparing hundreds/thousands of unique absorption/emission lines to model templates through crosscorrelation, we can highly boost the signal and constrain the chemical abundances, the planetary rotation, the projected equatorial wind, and even the temperaturepressure (T-P) profile of the atmosphere for emission spectroscopy data.

With the equilibrium temperature $\left(\mathrm{T}_{\text {eq }}\right)$ similar to M-dwarfs, similar prominent atomic/molecular opacity sources (Fe I, Fe II, Na I, and Ti II, TiO, VO, AlO, FeH, $\mathrm{CO}, \mathrm{H}_{2} \mathrm{O}$, and $\mathrm{OH}$ ) are expected to be found in the atmosphere of hot Jupiters $\left(\mathrm{T}_{\mathrm{eq}}<2200 \mathrm{~K}\right)$ and ultra hot Jupiters $\left(\mathrm{T}_{\text {eq }}>2200 \mathrm{~K}\right)$. Many of these optical opacity sources have been detected (e.g., Nugroho et al. 2017; von Essen et al. 2019; Hoeijmakers et al. 2019; Yan et al. 2019; Pino et al. 2020; Yan et al. 2021). In the nearinfrared, however, the most frequently detected species using high-resolution spectroscopy are $\mathrm{CO}$ and $\mathrm{H}_{2} \mathrm{O}$ (Snellen et al. 2010; De Kok et al. 2013; Birkby et al. 2013; Lockwood et al. 2014; Wang et al. 2018; Cabot et al. 2019; Webb et al. 2020). The opacities in this wavelength region play an important role as a coolant in the atmosphere and constraining their abundance would allow us to study the climate of unique planetary population and estimate the $\mathrm{C} / \mathrm{O}$ ratios thus inferring planetary formation history (Öberg et al. 2011). We, therefore, analysed the day-side spectrum of WASP-33b, one of the hottest ultra-hot Jupiters $\left(\mathrm{T}_{\text {day }}>3100 \mathrm{~K}\right.$, e.g., De Mooij et al. 2013) orbiting a fast-rotating $\delta$-scuti A5-type star (Collier Cameron et al. 2010), to search for molecular signatures in the near-infrared.

In this letter, we present the first detection of highresolution $\mathrm{OH}$ emission and the evidence of $\mathrm{H}_{2} \mathrm{O}$ emission in the day-side spectra of WASP-33b. In Section 2 , we describe the observations and data reduction. We then describe our modeling of the planetary emission spectrum in Section 3, and in Section 4, we detail our methodology in validating the accuracy of the $\mathrm{OH}$ linelists and searching the signal of $\mathrm{OH}$ and $\mathrm{H}_{2} \mathrm{O}$ in the atmosphere of the planet. Finally, in Section 5, we present and discuss our findings and their implications for the planetary atmosphere.

\section{OBSERVATIONS AND DATA REDUCTIONS}

We observed WASP-33 on the second half of the night of 30 September 2020 using the InfraRed Doppler instrument $(\mathrm{IRD} ; \mathrm{R} \approx 70,000 ; \lambda \approx 0.97-1.75 \mu \mathrm{m}$, Tamura et al. 2012; Kotani et al. 2018) on the Subaru 8.2-m telescope (PID: S20B-008, PI: S.K. Nugroho). We continuously observed the target with an exposure time of $300 \mathrm{~s}$ per frame without the laser frequency comb in natural guide star mode. The weather during the observations was not always stable, therefore we were only able to obtain 33 exposures covering the orbital phase of WASP-33b from $\approx 0.597$ to 0.700 (there were some gaps close to the middle of the observations due to clouds). We converted the Julian Date UTC to Barycentric Julian Date in Barycentric Dynamical Time (BJD ${ }_{\mathrm{TDB}}$ ) using the online calculator from Eastman et al. (2010) then calculated the orbital phase using the transit epoch taken from Johnson et al. (2015).

The data were reduced following Hirano et al. (2020) resulting in 70 spectral orders ranging from $\approx 9260-17419$ $\AA$ with an average signal-to-noise ratio $(\mathrm{S} / \mathrm{N})$ of 140 . We fitted the continuum of the spectrum with the highest average $\mathrm{S} / \mathrm{N}$ using CONTINUUM task in $\mathrm{IRAF}^{1}$ and divided it out from the data. Any possible blaze function variations were then corrected following the procedure in Nugroho et al. (2020b). Then, the sky emission lines, bad pixels, and regions were visually identified and masked. Additionally, we also masked any pixels that have a flux less than 10 percent of the continuum. In total, we masked 13.9 percent of the total number of pixels of the data. Finally, the spectra of each spectral order were aligned into 2-dimensional arrays with wave-

\footnotetext{
${ }^{1}$ The Image Reduction and Analysis Facility (IRAF) is distributed by the US National Optical Astronomy Observatories, operated by the Association of Universities for Research in Astronomy, Inc., under a cooperative agreement with the National Science Foundation.
} 
length along one axis and orbital phase along with the other. We estimated the uncertainty of each pixel by taking the outer product of the standard deviation of each wavelength and exposure bin, then normalized by the standard deviation of the whole array.

To check if there is any wavelength shift during the observation, the data were cross-correlated with the Doppler-shifted telluric templates produced using the Cerro Paranal Sky Model (Noll et al. 2012; Jones et al. 2013) over a velocity range of $-50 \mathrm{~km} \mathrm{~s}^{-1}$ to 50 $\mathrm{km} \mathrm{s}^{-1}$ in $0.01 \mathrm{~km} \mathrm{~s}^{-1}$ steps. We found no significant shift $\left(<0.05 \mathrm{~km} \mathrm{~s}^{-1}\right)$ compared to the precision that we need for this analysis, therefore we did not attempt to correct for this.

Before searching for any exoplanetary atomic or molecular signal using cross-correlation, we removed the telluric and stellar lines using a de-trending algorithm, SysRem (Tamuz et al. 2005), which has been successfully adopted for high-resolution Doppler spectroscopy (e.g., Birkby et al. 2013). SysRem fits the systematic trend in the wavelength bin direction which might be due to variation in airmass, water vapor column level, and others. Following Gibson et al. (2020), we run SysRem directly in flux for each spectral order independently. For each iteration, we summed the best-fit SysRem model and divided out from the data and the uncertainty array to propagate the error. Finally, any outliers more than five times the standard deviation of the residual were masked. A step-by-step overview of these procedures is shown in Figure 1. As in the previous analyses using SysRem (Gibson et al. 2020; Merritt et al. 2020; Nugroho et al. 2020b,a; Yan et al. 2020), instead of determining the optimal SysREM iteration of each order, we used the same number of iteration for all orders. The results are shown in Section 5 .

\section{PLANETARY EMISSION SPECTRUM TEMPLATES}

The planetary emission spectrum template was created by assuming 70 atmospheric layers evenly spaced in $\log$ pressure from $10^{2}$ to $10^{-8}$ bar of $1 \mathrm{D}$ plane-parallel hydro-static atmosphere, and a planetary-mass and radius of $3.266 \mathrm{M}_{\mathrm{J}}$ and $1.679 \mathrm{R}_{\mathrm{J}}$, respectively (Kovács et al. 2013). We adopted a thermally inverted T-P profile used in Nugroho et al. (2020a) which was calculated using the equation in Guillot (2010) assuming the visible mean opacity is twice the infrared mean opacity $\left(0.01 \mathrm{~cm}^{2} \mathrm{~g}^{-1}\right.$, e.g., dominated by $\mathrm{H}^{-}$opacity), an internal temperature of $100 \mathrm{~K}$ and $T_{\text {eq }}$ of $3100 \mathrm{~K}$ (assuming uniform day-side only re-radiation).

We produced 5 planetary spectrum models with single molecular opacity for $\mathrm{H}_{2} \mathrm{O}$ and $\mathrm{OH}$, with three and

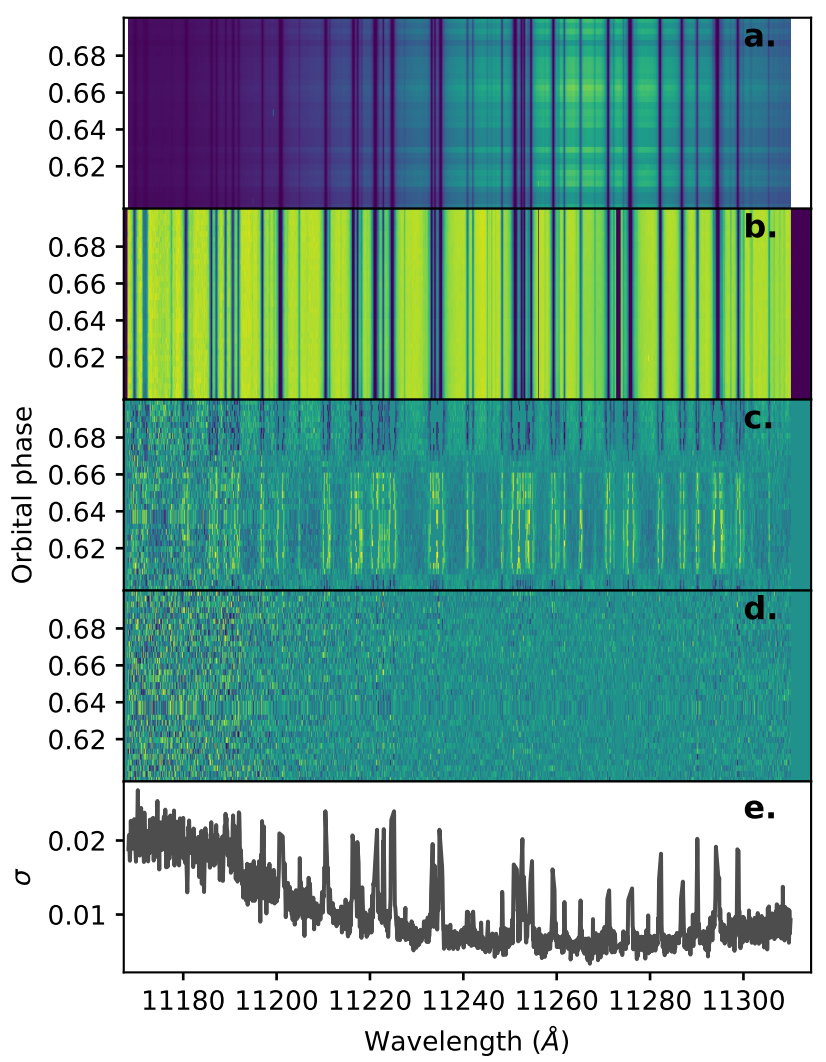

Figure 1. The example of step-by-steps of telluric and stellar line removal for order 26. (a.) The reduced spectra before normalizing and bad-pixels masking. (b.) The normalized reduced spectra after masking bad-pixels and pixels with a value less than 0.1 . (c.) The reduced spectra after dividing each wavelength bins by their mean values. (d.) The residual spectra after three iterations of SysRem. (e.) The standard deviation $(\sigma)$ of each wavelength bin in the residual spectra.

two different line-lists, respectively. The cross-sections of molecular species were computed using HELIOS-K (Grimm \& Heng 2015) at a resolution of $0.01 \mathrm{~cm}^{-1}$ from 9100 to 17800 Åassuming a Voigt line profile taking into account natural and thermal broadening only and a line wing cut-off of $100 \mathrm{~cm}^{-1}$. For $\mathrm{H}_{2} \mathrm{O}$, we used the linelist database of POKAZATEL (Polyansky et al. 2018), HITEMP 2010 (Rothman et al. 2010), and BT2 (Barber et al. 2006); for $\mathrm{OH}$, we used the updated linelist database of HITEMP (the updated HITEMP 2020, Rothman et al. 2010; Brooke et al. 2016; Yousefi et al. 2018; Noll et al. 2020) and MoLLIST (Brooke et al. 2016; Yousefi et al. 2018; Bernath 2020). For continuum opacity, we included the bound-free and free-free absorption of $\mathrm{H}^{-}$using the equation from John (1988), and collision-induced absorption (CIA) of $\mathrm{H}_{2}-\mathrm{H}_{2}$ (Abel et al. 2011) and $\mathrm{H}_{2}$-He (Abel et al. 2012). 
We used FAsTCHEM (Stock et al. 2018) to estimate the abundances of chemical species, and the mean molecular weight of each atmospheric layer assuming chemical equilibrium and solar $\mathrm{C} / \mathrm{O}$. We then produced the emission spectrum by solving the Schwarzchild equation following Nugroho et al. (2017, 2020a). We divided the resulting spectra by the flux of the star assuming a black body spectrum, $\mathrm{R}_{\star}$ of $1.509 \mathrm{R}_{\odot}$, and $T_{\text {eff }}$ of $7400 \mathrm{~K}$ then convolved with a Gaussian kernel to the spectral resolution of $\operatorname{IRD}^{2}$. Finally, we subtracted the planetary continuum from each model, which was determined by the continuum opacity of $\mathrm{CIA}$ and $\mathrm{H}^{-}$, estimated using a minimum filter with a window of $55 \AA$. The final result is the line contrast relative to the stellar continuum profile (see Figure 2).

\section{CROSS-CORRELATION AND LIKELIHOOD MAPPING}

\subsection{Accuracy of the Position of the Lines in the $\mathrm{OH}$ Line-lists}

In the cross-correlation analysis of high-resolution spectroscopy data, the accuracy of the template, therefore the line-list, has a crucial role in detecting chemical species. Using an incomplete or incorrect line-list might result in biased retrieved parameters (Brogi \& Line 2019), or a false-negative detection even when the chemical species exists (Flowers et al. 2019). Following previous analyses (e.g., Hoeijmakers et al. 2015; Nugroho et al. 2017), we investigated the accuracy of the position of the lines in the $\mathrm{OH}$ line-lists that we used by cross-correlating the $\mathrm{OH}$ planetary spectrum models with GJ 436 spectrum (M2.5V, $\mathrm{T}_{\text {eff }}=3416 \mathrm{~K}$, von Braun et al. 2012) that has a similar temperature as the day-side of WASP-33b. The cross-correlation was done using the Pearson cross-correlation equation over a range of velocity order-by-order. GJ 436's IRD data, taken during the engineering observations, were downloaded from SMOKA (Baba et al. 2002), and reduced in a similar way to our data. Since individual IRD frames are contaminated by telluric lines including air-glow $\mathrm{OH}$ emissions, we processed each spectrum and combined multiple frames taken on different epochs to disentangle the stellar lines from the telluric ones based on the procedure described in Hirano et al. (2020). We should note that the first five spectral orders of the GJ 436 spectrum were still heavily affected by telluric absorption, and thus not included in the cross-correlation analyses below.

\footnotetext{
${ }^{2}$ Using PYASL.INSTRBROADGAUSSFAST
}

The cross-correlation functions of both line-lists are similar in strength and located at the expected radial velocity indicating that they are accurate (see Figure 5 in the Appendix). Most of the lines are located in the $H$-band $(\lambda>1.460 \mu \mathrm{m})$ as was expected for an earlytype M-dwarf and our spectrum template (see Figure 2). Meanwhile, in the $Y$ - and $J$-band, there are no significant or weak correlations which most likely due to weak $\mathrm{OH}$ absorption lines in the GJ 436 spectrum. With this result, we concluded that the position of the lines in both line-lists is accurate enough for our purpose.

\subsection{Searching for $\mathrm{OH}$ and $\mathrm{H}_{2} \mathrm{O}$ signatures}

Even after removing the telluric and stellar lines, the planetary signal is expected to be still buried under the noise. As there are many resolved lines of $\mathrm{H}_{2} \mathrm{O}$ and $\mathrm{OH}$ in the IRD wavelength range, we combined them to boost the planetary signal by cross-correlating the residual of each SysREM iterations with the Dopplershifted planetary spectrum templates from $-500 \mathrm{~km} \mathrm{~s}^{-1}$ to $+500 \mathrm{~km} \mathrm{~s}^{-1}$ in $1 \mathrm{~km} \mathrm{~s}^{-1}$ steps following:

$$
\operatorname{CCF}(v)=\sum_{i} \frac{f_{i} m_{i}(v)}{\sigma_{i}^{2}}
$$

where $f_{i}$ is the mean-subtracted data, $m_{i}$ is the meansubtracted spectrum model Doppler-shifted to a radial velocity of $v, \sigma_{i}^{2}$ is the variance at $i$ th wavelength bin. We performed this for each spectral order and summed them excluding the spectral order that has significant telluric removal residuals (spectral order with central wavelength of $13529.12,14317.24$ and $14457.64 \AA$ ).

We calculated orbital velocity-systemic velocity $\left(K_{\mathrm{p}}-\mathrm{v}_{\mathrm{sys}}\right)$ map by shifting the cross-correlation functions (CCFs) to the planetary rest-frame over a range of $K_{\mathrm{p}}$, from 0 to $+300 \mathrm{~km} \mathrm{~s}^{-1}$, and $v_{\text {sys }}$, from $-125 \mathrm{~km} \mathrm{~s}^{-1}$ to $+125 \mathrm{~km} \mathrm{~s}^{-1}$, both in $0.2 \mathrm{~km} \mathrm{~s}^{-1}$ steps using linear interpolation and summed over time. The radial velocity of the planet at a given orbital phase $(\phi), \mathrm{RV}_{\mathrm{p}}(\phi)$, assuming the planet has a circular orbit is

$$
\mathrm{RV}_{\mathrm{p}}(\phi)=K_{\mathrm{p}} \sin (2 \pi \phi)+v_{\mathrm{sys}}+v_{\text {bary }}
$$

where $v_{\text {bary }}$ is the barycentric correction ${ }^{3}$, and $\phi$ is the orbital phase of the planet.

From Collier Cameron et al. (2010); Nugroho et al. (2017); Yan et al. (2019); Nugroho et al. (2020a), the planet signal is expected at $K_{\mathrm{p}}$ of $\approx 230 \mathrm{~km} \mathrm{~s}^{-1}$ and $v_{\text {sys }}$ of $\approx-3 \mathrm{~km} \mathrm{~s}^{-1}$. We computed the $\mathrm{S} / \mathrm{N}$ by dividing the $K_{\mathrm{p}}-\mathrm{v}_{\mathrm{sys}}$ map by its standard deviation calculated by

\footnotetext{
3 using PYASL.HELCORR
} 


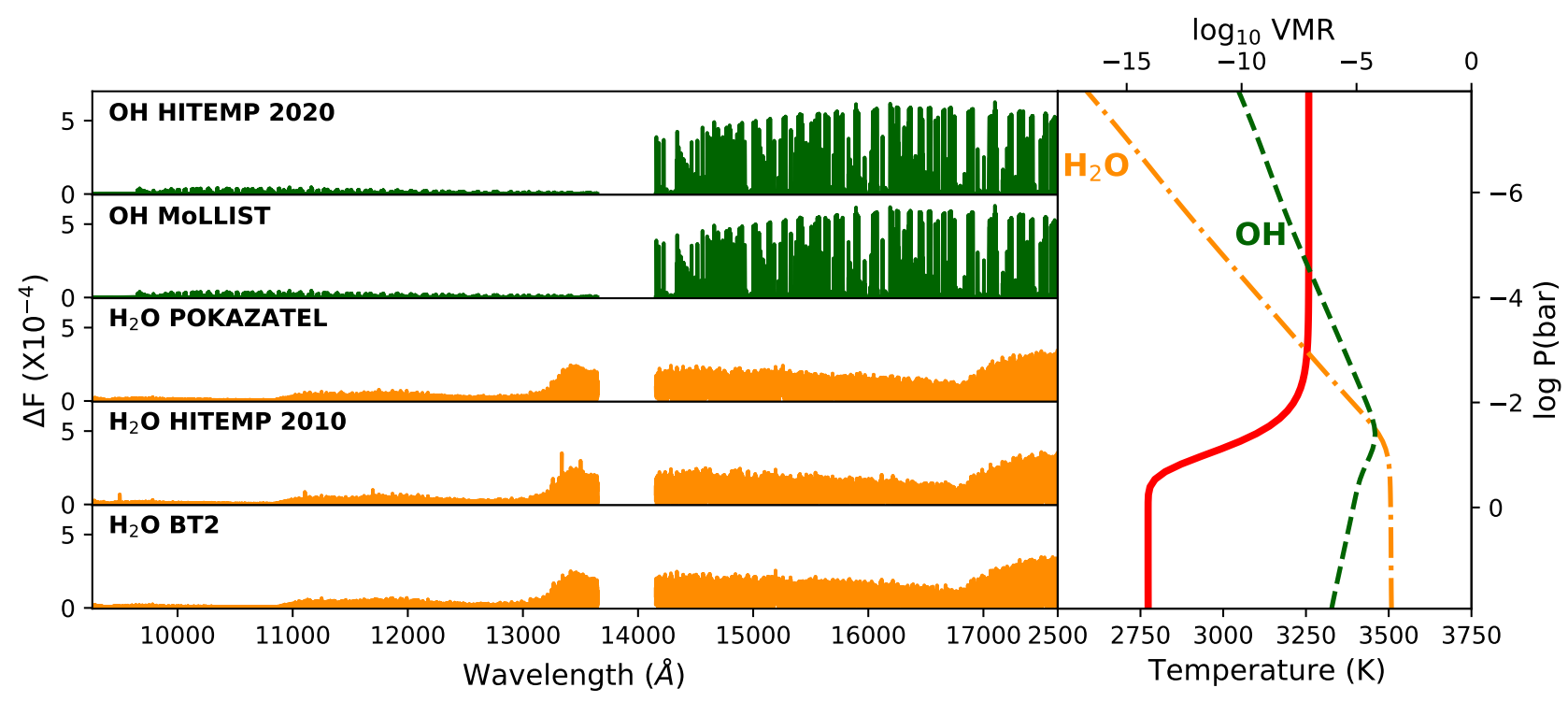

Figure 2. left panel: The normalized planetary spectrum models for $\mathrm{OH}$ and $\mathrm{H}_{2} \mathrm{O}$ using different line-lists. The scale on the $\mathrm{y}$-axis is the same for all panels, therefore the strength of the emission lines can be compared visually. right panel: Temperaturepressure profile of WASP-33b that was adopted in the modeling (red lines). The chemical equilibrium abundances (in volume mixing ratio, VMR) calculated using FASTCHEM are indicated by dark green dashed-line for OH and dark orange dotted-line for $\mathrm{H}_{2} \mathrm{O}$.

avoiding the area $\pm 25 \mathrm{~km} \mathrm{~s}^{-1}$ from the expected planet signal.

Next, we converted the cross-correlation map to a likelihood map $(\mathcal{L})$ using the $\beta$-optimised likelihood function following Gibson et al. (2020, see also Brogi \& Line 2019):

$\ln \mathcal{L}=-\frac{N}{2} \ln \left[\frac{1}{N}\left(\sum \frac{f_{i}^{2}}{\sigma_{i}^{2}}+\alpha^{2} \sum \frac{m_{i}^{2}}{\sigma_{i}^{2}}-2 \alpha \mathrm{CCF}\right)\right]$,

where $\alpha$ is the scale factor of the model and $N$ is the total number of pixels. A 3-dimensional likelihood or posterior data-cube (assuming uniform priors) was then produced with a range of $\alpha$ from 0.01 to 1.50 in 0.01 steps by subtracting the global maximum value from the cube and calculating the exponential, this normalized the likelihood to 1 . We then marginalized it by summing the maps over parameters to get the best-fit parameters and uncertainties. We estimated the significance of detection by dividing the median value of the conditional distribution of $\alpha$ at the best-fit value of $K_{\mathrm{p}}$ and $v_{\text {sys }}$ by its uncertainty.

\section{RESULTS}

\subsection{OH emission in the day-side of WASP-33b}

We detected the $\mathrm{OH}$ emission signature at $\mathrm{S} / \mathrm{N}$ of 5.4 and significance of $\mathbf{5 . 5} \sigma$ at $K_{\mathrm{p}}$ of $230.9_{-7.4}^{+6.9} \mathrm{~km} \mathrm{~s}^{-1}$ and $v_{\mathrm{sys}}$ of $-0.3_{-5.6}^{+5.3} \mathrm{~km} \mathrm{~s}^{-1}$ (see Figure $3 \mathrm{~b}$ and 4 ) consistent with previous results although with larger uncertainties due to narrower orbital phase coverage (e.g., Nugroho et al. 2017, 2020a; Yan et al. 2019). From Figure 3a, the planet signal appears as a bright stripe shown by the white arrows. The strength of the signal varied with time which might be due to the unstable weather during the observation that potentially affects the telluric removal using SysRem. The $\alpha$ is constrained to $0.47 \pm 0.09$, which means that we have overestimated the strength of the signal. This could be due to the effect of SYsREM which might have eroded and altered the observed exoplanet signal. Furthermore, the inhomogeneity of the day-side of WASP-33b or the overestimation of the T-P profile and/or the $\mathrm{OH}$ abundance in the modeling (photo-dissociation and other possible overlapping opacity sources) could also be the cause. We leave a more detailed analysis to future works.

For both line-lists, the detected signals become prominent after two SysRem iteration and at their highest $\mathrm{S} / \mathrm{N}$ after three iterations before getting weaker with more iterations. The cross-correlation maps, the $\mathrm{S} / \mathrm{N}$ and/or detection significance, and the constraint on alpha for both templates are the same, therefore, we show the result for OH HITEMP 2020 only. The only difference of the result using the two line-lists is in the velocity constraint which differs by $\approx 0.1 \mathrm{~km} \mathrm{~s}^{-1}$. This is expected as the two line-lists are based on the same data, although OH HITEMP 2020 line-list was just recently updated based on the observed high-resolution OH telluric lines (Noll et al. 2020). 


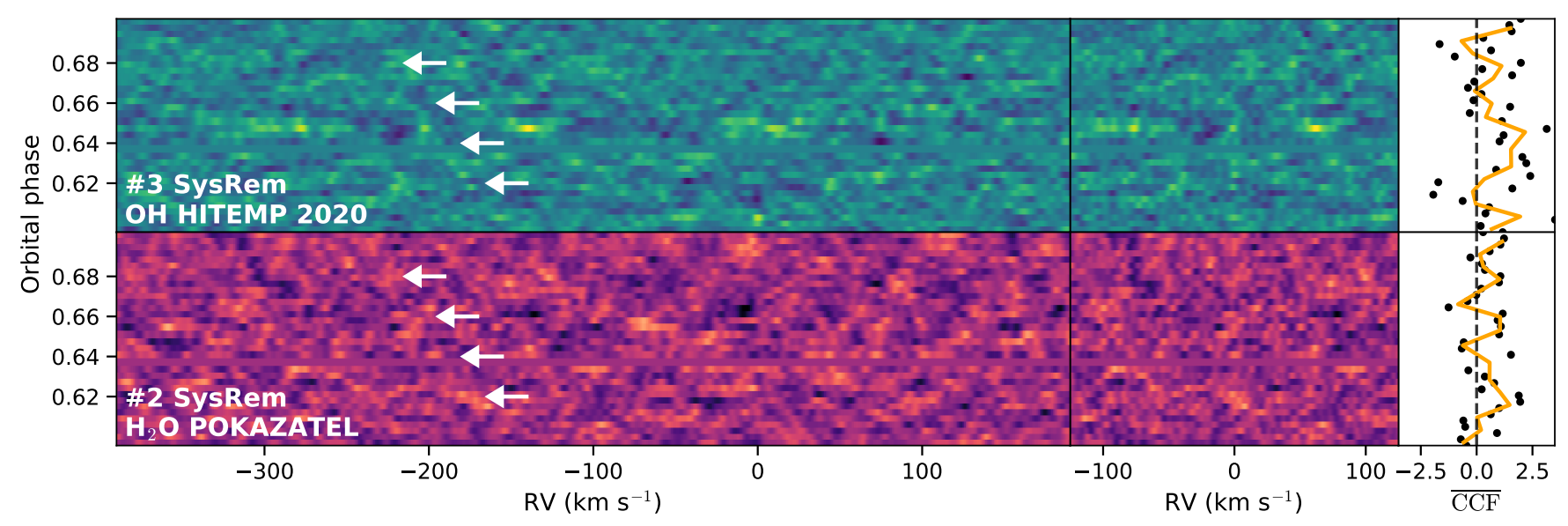

(a)

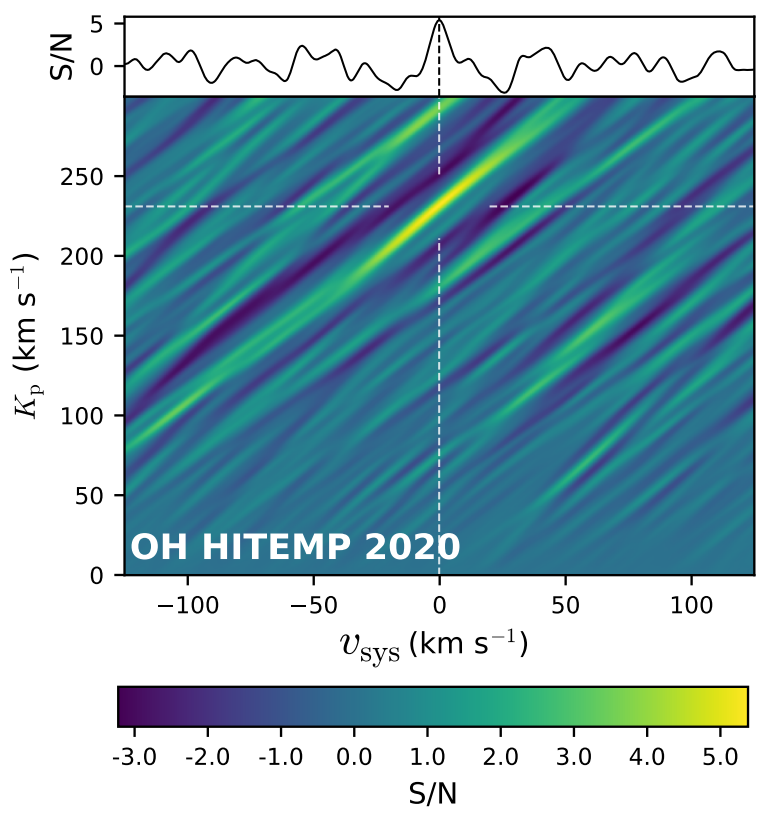

(b)

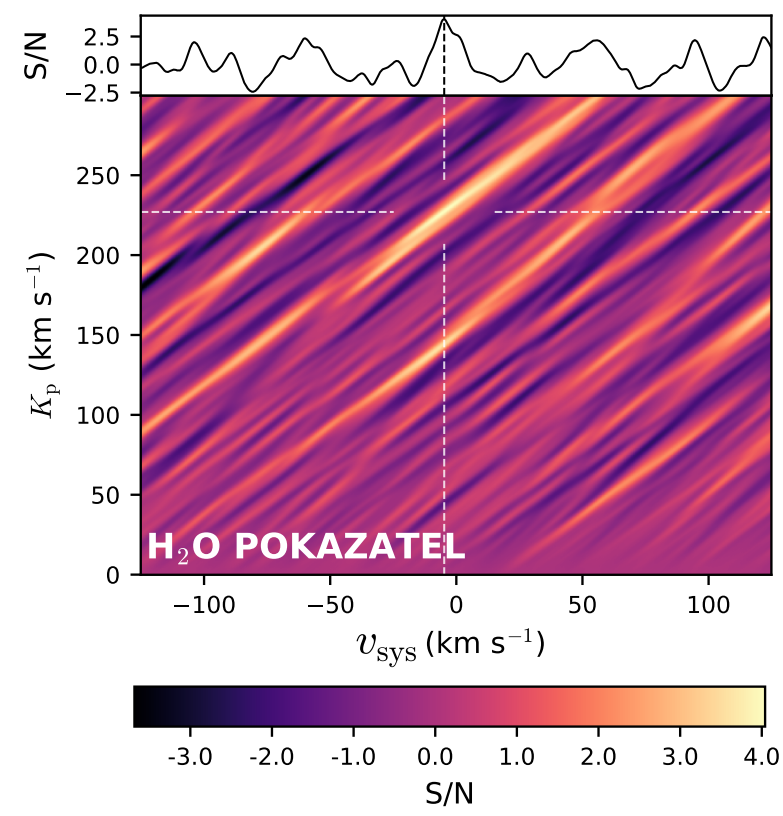

(c)

Figure 3. The cross-correlation results after three SysREM iteration for OH HITEMP 2020 (upper panel of a) and after two SYSREM iteration for $\mathrm{H}_{2} \mathrm{O}$ POKAZATEL (lower panel of a). left panel: The cross-correlation map at the telluric rest-frame. The planetary signal appears as a bright diagonal stripe and is shown by the white arrows. middle panel: The CCFs at the planetary rest-frame. right panel: The mean CCFs of $\pm 3 \mathrm{~km} \mathrm{~s}^{-1}$ from the center of the planet signal are shown by black dots. The orange line shows the binned-CCFs by two exposures. The black dashed line indicates the zero value. The $K_{\mathrm{p}}-\mathrm{v}_{\mathrm{sys}}$ map for the OH HITEMP 2020 (b) and $\mathrm{H}_{2} \mathrm{O}$ POKAZATEL (c). The white dashed line indicates the maximum signal on the map. The upper panels show the CCFs at the $K_{\mathrm{p}}$ of $230.9 \mathrm{~km} \mathrm{~s}^{-1}$ and $227.5 \mathrm{~km} \mathrm{~s}^{-1}$ for OH HITEMP 2020 and $\mathrm{H}_{2} \mathrm{O}$ POKAZATEL, respectively. The color-bar shows the $\mathrm{S} / \mathrm{N}$ of the $K_{\mathrm{p}}-\mathrm{v}_{\text {sys }}$ map. 


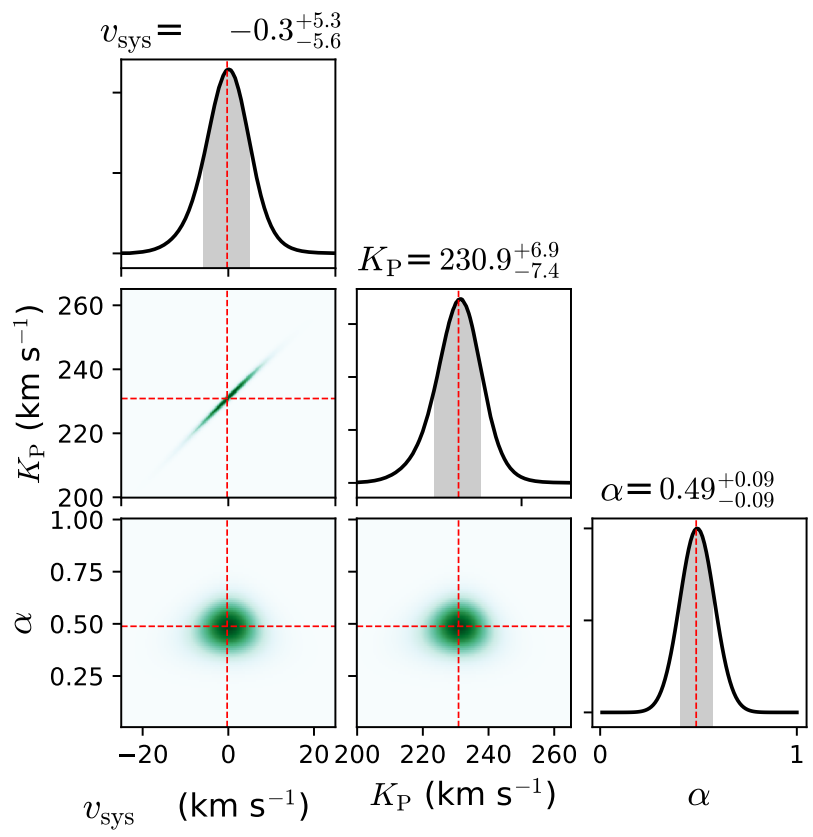

Figure 4. The marginalized likelihood distribution of $K_{\mathrm{p}}$ and $\mathrm{v}_{\mathrm{sys}}$, and conditional likelihood distribution of $\alpha$ at the best-fit $K_{\mathrm{p}}$ and $\mathrm{v}_{\mathrm{sys}}$ for OH HITEMP 2020. The red dashed lines show the median value of the corresponding distribution. The gray shaded area indicates the $\pm 1 \sigma$ limit from the median value.

Compared to other ultra-hot Jupiters, the atmosphere of WASP-33b's atmosphere is more difficult to characterise with low-resolution spectroscopy/photometry due to the $\delta$-Scuti pulsations of its host star. These pulsations can also affect high-spectral resolution searches for atomic species that occur both in the stellar photosphere and in the planet's atmosphere, such as Fe I (Nugroho et al. 2020a, Herman et al. in prep.) where a region of $\sim \pm v_{\text {rot* }} \sin i$ has to be excluded from analysis, as the stellar pulsations overlap with the signal from the planet. Since $\mathrm{OH}$ is not present in the stellar atmosphere of an A-star, this does not pose a problem for the results presented in this paper. In addition, the lack of signal at $K_{\mathrm{p}}$ of $0 \mathrm{~km} \mathrm{~s}^{-1}$ or RV of $0 \mathrm{~km} \mathrm{~s}^{-1}$ indicates that there is no contamination from telluric $\mathrm{OH}$ emission. Furthermore, as the trail of the signal appeared only at the expected planetary velocity (see Figure 3a and $\mathrm{b}$ ), we are confident that the detected $\mathrm{OH}$ emission signature is originating from the exoplanet.

\subsection{Marginal detection of weak $\mathrm{H}_{2} \mathrm{O}$ emission?}

On the other hand, we only marginally detected $\mathrm{H}_{2} \mathrm{O}$ emission in the H-band at $K_{\mathrm{p}}$ of $227.5_{-8.5}^{+8.7} \mathrm{~km} \mathrm{~s}^{-1}$ and $v_{\text {sys }}$ of $-4.3_{-6.2}^{+7.5} \mathrm{~km} \mathrm{~s}^{-1}$ using POKAZATEL line-list at $\mathrm{S} / \mathrm{N}$ of 4.0 and significance of $5.2 \sigma$. This is consistent with the prediction that most of the molecular feature in the $Y$ - and $J$-band is muted by $\mathrm{H}^{-}$opacity (Arcangeli et al. 2018; Parmentier et al. 2018). The S/N of the detected signal may be different to the significance estimated from the conditional likelihood distribution of alpha as they both use different methods to evaluate the noise. For example with the $\mathrm{S} / \mathrm{N}$ method we have to define a region around the peak to compute the standard deviation, where the conditional likelihood compares to a null signal at the same $K_{\mathrm{p}}$ and $\mathrm{v}_{\mathrm{sys}}$. In this case the lower $\mathrm{S} / \mathrm{N}$ is likely due to bright spots in the $K_{\mathrm{p}}-\mathrm{v}_{\mathrm{sys}}$ map, and the calculated $\mathrm{S} / \mathrm{N}$ changes with the arbitrary choice of region used to compute the noise. We argue that the conditional likelihood method is more principled and less arbitrary, but it is nonetheless beneficial to compute the detection significance in multiple ways.

The detected signal is the strongest after two SYsRem iterations instead of three (see Figure 3c) although it only differs by 0.1 from after two to four SysRem iterations. In the $\mathrm{OH}$ templates, most of the strongest lines are distributed in the middle of the $H$-band, while for $\mathrm{H}_{2} \mathrm{O}$, the strong lines are distributed more in both edges of the $H$-band and the redder edge of the $Y$-band. As we performed SysRem order-by-order independently, the "optimum" number of SYsREM iteration for each order (i.e. optimally removes the telluric lines and leave the planetary signals mostly intact) are potentially different (e.g., Sánchez-López et al. 2019). Therefore, the number of SYSREM iterations that result in the highest $\mathrm{S} / \mathrm{N}$ of $\mathrm{H}_{2} \mathrm{O}$ signal is potentially to be different than that for the $\mathrm{OH}$ signal.

As for HITEMP 2010, the signal around the same location is much weaker (see Figure 6). As we are probing the day-side of the planet, the analysis is sensitive to the T-P profile of the atmosphere. Therefore, following Nugroho et al. (2020a), we cross-correlated the data with a range of different $\mathrm{H}_{2} \mathrm{O}$ VMR templates assuming uniform abundance with the altitudes to probe different temperatures while minimizing free parameter. However, we found no significant improvement more than our previous analysis. We also tried using BT2 line-list but found no signal (see Figure 6).

Gandhi et al. (2020) also marginally detected $\mathrm{H}_{2} \mathrm{O}$ absorption in the $K$-band from the day-side of HD 179949 using model produced with POKAZATEL linelist $(\mathrm{S} / \mathrm{N} \approx 4.1)$ and at $\mathrm{S} / \mathrm{N}$ of 3.2 using HITEMP 2010. For colder hot Jupiter like HD 189733 b, however, both line-lists produced similar detection. These different detections of $\mathrm{H}_{2} \mathrm{O}$ signal could be caused by different line positions and strengths, and the completeness of the line-lists. Thus, even when assuming the same T-P profile and chemical abundances, the results can be different (Brogi \& Line 2019). Moreover, at high temperatures, 
the weak lines can be as abundant as the strong lines thus the completeness of the line-list can be as important as the accuracy of the strong lines. To confirm this, more observational data are needed as we were only able to obtain a marginal detection.

Lastly, we performed an injection test at $K_{\mathrm{p}}$ of $227.5 \mathrm{~km} \mathrm{~s}^{-1}$ and $v_{\mathrm{sys}}$ of $-4.3 \mathrm{~km} \mathrm{~s}^{-1}$ with the model produced using the T-P profile and the lower limit of the uniform chemical abundance of $\mathrm{H}_{2} \mathrm{O}$ retrieved by Haynes et al. (2015), re-ran the SysRem then cross-correlated with the template. In contrast to our marginal detection, we were able to recover the injected signal with high significance $(\mathrm{S} / \mathrm{N}>6)$. Regardless of whether our detected signal is real or not, this indicates that the retrieved $\mathrm{H}_{2} \mathrm{O}$ abundance and/or the T-P profile in Haynes et al. (2015) might have been overestimated for the upper atmosphere which could be due to the exclusion of the thermal-dissociation effect or $\mathrm{OH} / \mathrm{H}^{-}$opacity in the retrieval.

\section{DISCUSSION AND CONCLUSION}

While the signature of $\mathrm{OH}$ has been detected in the atmosphere of Earth, the Saturn magnetosphere, Venus, and Mars (Meinel 1950; Shemansky et al. 1993; Piccioni et al. 2008; Todd Clancy et al. 2013), this is the first time that its signature has been detected in the atmosphere of an exoplanet. Along with $\mathrm{O}, \mathrm{OH}$ is one of the most important radical species that drive atmospheric chemistry. For a hot Jupiter like HD 209458b, OH is mainly produced from the photolysis of $\mathrm{H}_{2} \mathrm{O}$ by the stellar UV (Liang et al. 2003). However, for a much hotter planet like WASP-33b, the thermochemical reaction is expected to be the dominant source of $\mathrm{OH}$ as the atmosphere is closer to thermochemical equilibrium (Visscher et al. 2006). Our result, which only marginally detected weak emission of $\mathrm{H}_{2} \mathrm{O}$, indicates that most of the $\mathrm{H}_{2} \mathrm{O}$ in the upper atmosphere is thermally dissociated consistent with the theoretical predictions (Parmentier et al. 2018). Thus, $\mathrm{OH}$ is expected to be one of the most dominant O-bearing molecules along with $\mathrm{CO}$ and should be considered when analyzing the emission spectrum of ultra-hot Jupiters, as well as search for in other planetary atmosphere.

Through an injection test, were $\mathrm{H}_{2} \mathrm{O}$ present in the day-side of WASP-33b at the abundance and temperature that were retrieved by Haynes et al. (2015), we would detect it at high-significance. As low-resolution spectroscopy probes a relatively deeper atmospheric layer than high-resolution spectroscopy, the retrieved parameters might not provide a reliable measurement for the upper atmosphere. Moreover, when there are overlapping unresolved features from multiple species, the retrieved parameters would be incorrect if the model does not consider all of the possible chemical species. Thus, combining low-resolution spectroscopy and highresolution spectroscopy would be required to get a more accurate and precise characterization of the exoplanet atmosphere (Brogi \& Line 2019; Gandhi et al. 2019). Finally, this work demonstrates the capability of IRD in characterizing the atmosphere of an exoplanet and its potential to complement the space-borne facilities (e.g., HST, JWST).

\section{ACKNOWLEDGMENTS}

We are extremely grateful to the anonymous referee for constructive and insightful comments that greatly improved the quality of this letter. This work is based on data collected at Subaru Telescope, which is operated by the National Astronomical Observatory of Japan. Our data reductions benefited from (PyRAF and) PyFITS that are the products of the Space Telescope Science Institute, which is operated by AURA for NASA. The M-dwarfs spectra are based on data collected at Subaru Telescope and obtained from the SMOKA, which is operated by the Astronomy Data Center, National Astronomical Observatory of Japan. H.K. is supported by a Grant-in-Aid from JSPS (Japan Society for the Promotion of Science), Nos. JP18H04577, JP18H01247, and JP20H00170. This work was also supported by the JSPS Core-to-Core Program "Planet" ${ }^{2}$ " and SATELLITE Research from Astrobiology Center (AB022006). N. P. G. gratefully acknowledges support from Science Foundation Ireland and the Royal Society in the form of a University Research Fellowship. T.H. acknowledges support from JSPS KAKENHI grant number 19K14783. Y.K. is supported by Special Postdoctoral Researcher Program at RIKEN. C.A.W. would like to acknowledge support from UK Science Technology and Facility Council grant ST/P000312/1. M.T. would like to acknowledge support from MEXT/JSPS KAKENHI grant Nos. 18H05442, 15H02063, and 22000005. M.I. acknowledges support from JSPS KAKENHI grant numbers 19J11805. JLB acknowledges funding from the European Research Council (ERC) under the European Union's Horizon 2020 research and innovation program under grant agreement No 805445. MB acknowledges support from the UK Science and Technology Facilities Council (STFC) research grant ST/S000631/1. We are also grateful to the developers of the NuMPY, Scipy, Matplotlib, Jupyter Notebook, and AsTROPY packages, which were used extensively in this work (Virtanen et al. 2020; Hunter 2007; Kluyver et al. 2016; Astropy Collaboration et al. 2013; Price-Whelan et al. 2018). 


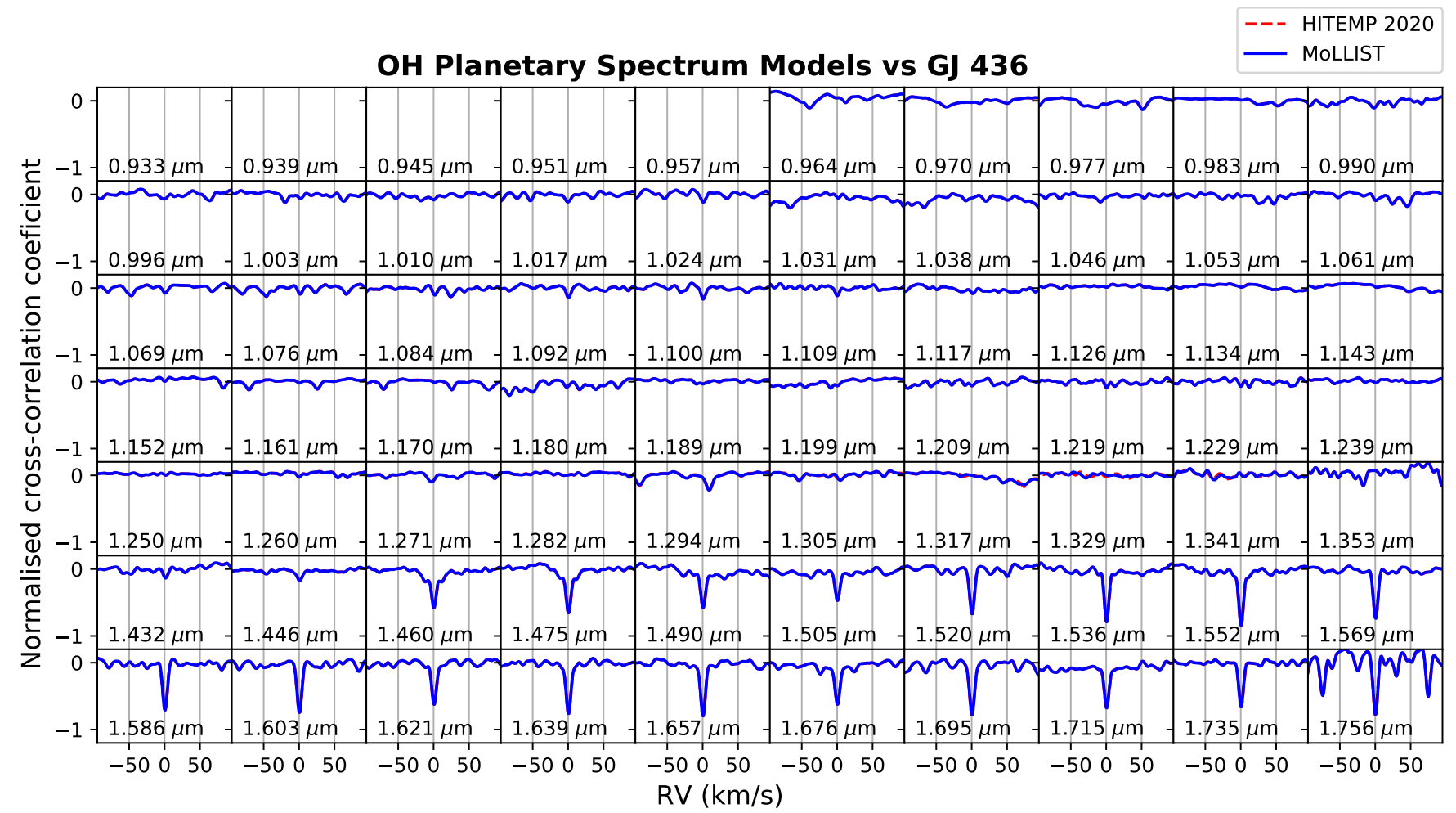

Figure 5. The CCFs between GJ 436 spectrum and OH planet spectrum models using HITEMP 2020 (red dashed-line) and MoLLIST (blue line) for each spectral order (each panel labeled by the median wavelength value of each spectral orders). The CCFs of HITEMP 2020 overlap with the CCFs of MoLLIST indicating that they have the similar accuracy.

\section{APPENDIX}

\section{A. ADDITIONAL FIGURES}

\section{REFERENCES}

Abel, M., Frommhold, L., Li, X., \& Hunt, K. L. C. 2011, Journal of Physical Chemistry A, 115, 6805, doi: 10.1021/jp109441f

—. 2012, JChPh, 136, 044319, doi: 10.1063/1.3676405

Arcangeli, J., Désert, J.-M., Line, M. R., et al. 2018, ApJL, 855, L30, doi: 10.3847/2041-8213/aab272

Astropy Collaboration, Robitaille, T. P., Tollerud, E. J., et al. 2013, A\&A, 558, A33, doi: 10.1051/0004-6361/201322068

Baba, H., Yasuda, N., Ichikawa, S.-I., et al. 2002, in Astronomical Society of the Pacific Conference Series, Vol. 281, Astronomical Data Analysis Software and Systems XI, ed. D. A. Bohlender, D. Durand, \& T. H. Handley, 298
Barber, R. J., Tennyson, J., Harris, G. J., \& Tolchenov, R. N. 2006, MNRAS, 368, 1087, doi: 10.1111/j.1365-2966.2006.10184.x

Bernath, P. F. 2020, JQSRT, 240, 106687, doi: 10.1016/j.jqsrt.2019.106687

Birkby, J. L., de Kok, R. J., Brogi, M., et al. 2013, MNRAS, 436, L35, doi: 10.1093/mnrasl/slt107

Brogi, M., \& Line, M. R. 2019, AJ, 157, 114, doi: $10.3847 / 1538-3881 /$ aaffd3

Brooke, J. S. A., Bernath, P. F., Western, C. M., et al. 2016, JQSRT, 168, 142, doi: 10.1016/j.jqsrt.2015.07.021

Cabot, S. H. C., Madhusudhan, N., Hawker, G. A., \& Gandhi, S. 2019, MNRAS, 482, 4422, doi: 10.1093/mnras/sty2994

Collier Cameron, A., Guenther, E., Smalley, B., et al. 2010, MNRAS, 407, 507, doi: 10.1111/j.1365-2966.2010.16922.x 


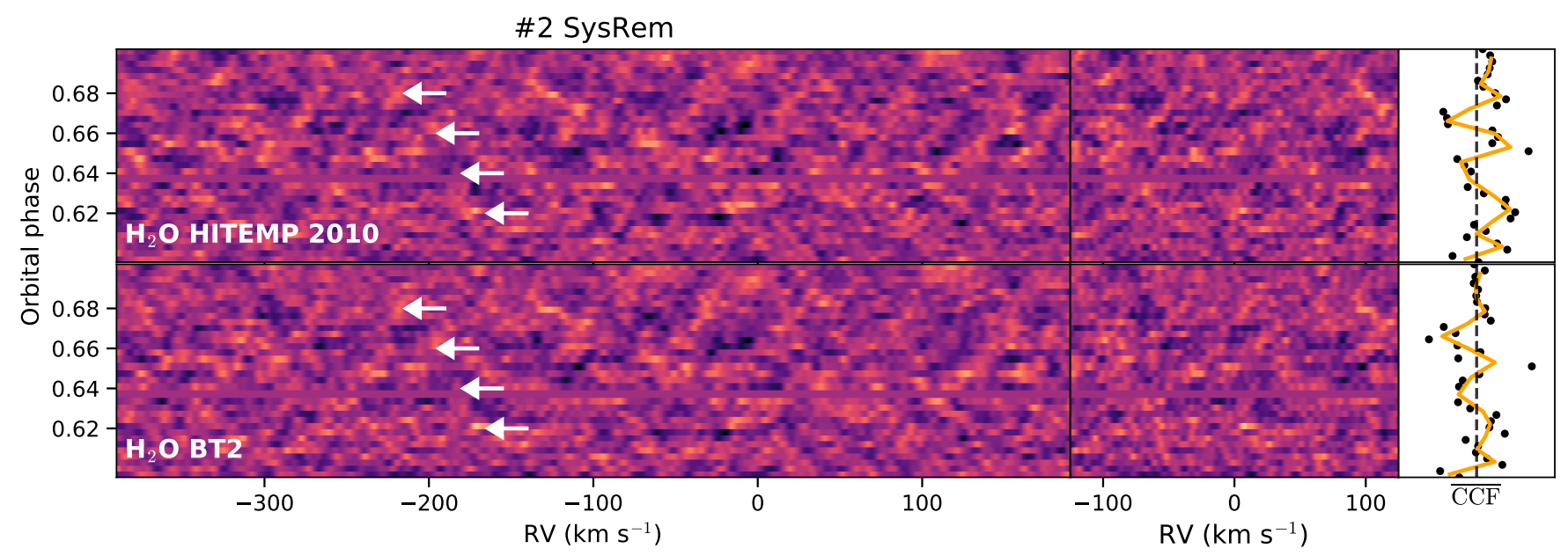

(a)
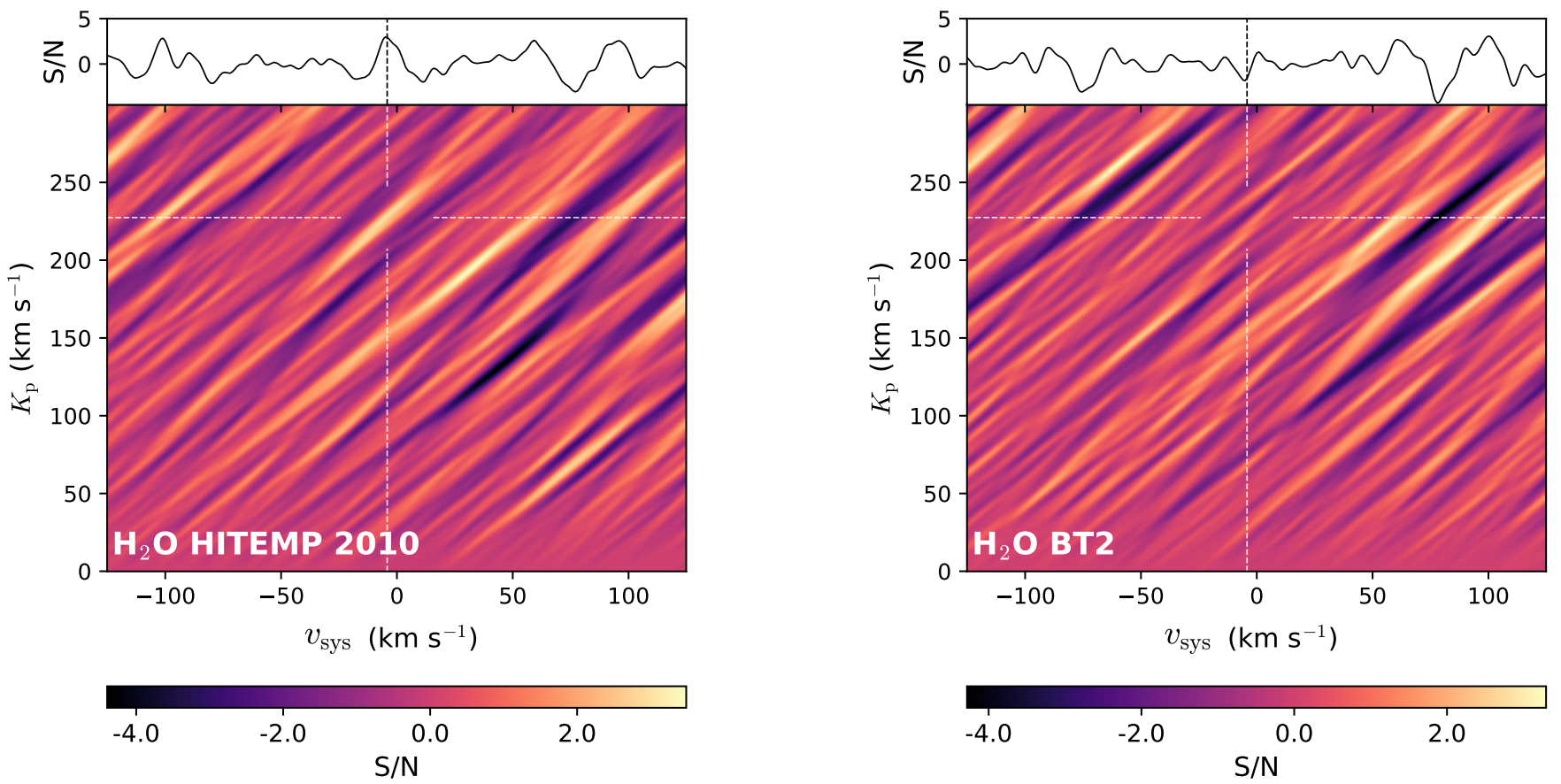

(b)

Figure 6. Similar to Figure 3 but for HITEMP 2010 and BT2. The white dashed line is fixed at the $K_{\mathrm{p}}$ of $227.5 \mathrm{~km} \mathrm{~s}^{-1}$ and $v_{\text {sys }}$ of $-4.3 \mathrm{~km} \mathrm{~s}^{-1}$. The upper panels show the CCFs at the same $K_{\mathrm{p}}$.

De Kok, R. J., Brogi, M., Snellen, I. A. G., et al. 2013,

A\&A, 554, A82, doi: 10.1051/0004-6361/201321381

De Mooij, E. J. W., Brogi, M., de Kok, R. J., et al. 2013,

A\&A, 550, A54, doi: 10.1051/0004-6361/201219434

Eastman, J., Siverd, R., \& Gaudi, B. S. 2010, PASP, 122, 935, doi: $10.1086 / 655938$
Flowers, E., Brogi, M., Rauscher, E., Kempton, E. M. R., \& Chiavassa, A. 2019, AJ, 157, 209, doi: $10.3847 / 1538-3881 / a b 164 c$

Gandhi, S., Madhusudhan, N., Hawker, G., \& Piette, A. 2019, arXiv e-prints, arXiv:1910.14042. https://arxiv.org/abs/1910.14042

Gandhi, S., Brogi, M., Yurchenko, S. N., et al. 2020, MNRAS, 495, 224, doi: 10.1093/mnras/staa981 
Gibson, N. P., Merritt, S., Nugroho, S. K., et al. 2020, MNRAS, 493, 2215, doi: 10.1093/mnras/staa228

Grimm, S. L., \& Heng, K. 2015, The Astrophysical Journal, 808, 182, doi: 10.1088/0004-637X/808/2/182

Guillot, T. 2010, A\&A, 520, A27, doi: 10.1051/0004-6361/200913396

Haynes, K., Mandell, A. M., Madhusudhan, N., Deming, D., \& Knutson, H. 2015, ApJ, 806, 146, doi: 10.1088/0004-637X/806/2/146

Hirano, T., Kuzuhara, M., Kotani, T., et al. 2020, PASJ, 72, 93, doi: 10.1093/pasj/psaa085

Hoeijmakers, H. J., de Kok, R. J., Snellen, I. A. G., et al. 2015, A\&A, 575, A20, doi: 10.1051/0004-6361/201424794

Hoeijmakers, H. J., Ehrenreich, D., Kitzmann, D., et al. 2019, A\&A, 627, A165, doi: 10.1051/0004-6361/201935089

Hunter, J. D. 2007, Computing in Science \& Engineering, 9, 90, doi: 10.1109/MCSE.2007.55

John, T. L. 1988, A\&A, 193, 189

Johnson, M. C., Cochran, W. D., Collier Cameron, A., \& Bayliss, D. 2015, ApJL, 810, L23, doi: 10.1088/2041-8205/810/2/L23

Jones, A., Noll, S., Kausch, W., Szyszka, C., \& Kimeswenger, S. 2013, A\&A, 560, A91, doi: 10.1051/0004-6361/201322433

Kluyver, T., Ragan-Kelley, B., Pérez, F., et al. 2016, in Positioning and Power in Academic Publishing: Players, Agents and Agendas, ed. F. Loizides \& B. Schmidt, IOS Press, $87-90$

Kotani, T., Tamura, M., Nishikawa, J., et al. 2018, in Society of Photo-Optical Instrumentation Engineers (SPIE) Conference Series, Vol. 10702, Ground-based and Airborne Instrumentation for Astronomy VII, ed. C. J. Evans, L. Simard, \& H. Takami, 1070211, doi: $10.1117 / 12.2311836$

Kovács, G., Kovács, T., Hartman, J. D., et al. 2013, A\&A, 553, A44, doi: 10.1051/0004-6361/201219642

Liang, M.-C., Parkinson, C. D., Lee, A. Y. T., Yung, Y. L., \& Seager, S. 2003, ApJL, 596, L247, doi: 10.1086/379314

Lockwood, A. C., Johnson, J. A., Bender, C. F., et al. 2014, ApJL, 783, L29, doi: 10.1088/2041-8205/783/2/L29

Meinel, I. A. B. 1950, ApJ, 111, 555, doi: 10.1086/145296

Merritt, S. R., Gibson, N. P., Nugroho, S. K., et al. 2020, arXiv e-prints, arXiv:2002.02795.

https://arxiv.org/abs/2002.02795

Noll, S., Kausch, W., Barden, M., et al. 2012, A\&A, 543, A92, doi: 10.1051/0004-6361/201219040

Noll, S., Winkler, H., Goussev, O., \& Proxauf, B. 2020, Atmospheric Chemistry \& Physics, 20, 5269, doi: 10.5194/acp-20-5269-2020
Nugroho, S. K., Gibson, N. P., de Mooij, E. J. W., et al. 2020a, ApJL, 898, L31, doi: 10.3847/2041-8213/aba4b6

—. 2020b, MNRAS, 496, 504, doi: 10.1093/mnras/staa1459

Nugroho, S. K., Kawahara, H., Masuda, K., et al. 2017, AJ, 154, 221, doi: 10.3847/1538-3881/aa9433

Öberg, K. I., Murray-Clay, R., \& Bergin, E. A. 2011, ApJL, 743, L16, doi: 10.1088/2041-8205/743/1/L16

Parmentier, V., Line, M. R., Bean, J. L., et al. 2018, A\&A, 617, A110, doi: 10.1051/0004-6361/201833059

Piccioni, G., Drossart, P., Zasova, L., et al. 2008, A\&A, 483, L29, doi: 10.1051/0004-6361:200809761

Pino, L., Désert, J.-M., Brogi, M., et al. 2020, ApJL, 894, L27, doi: 10.3847/2041-8213/ab8c44

Polyansky, O. L., Kyuberis, A. A., Zobov, N. F., et al. 2018, MNRAS, 480, 2597, doi: 10.1093/mnras/sty1877

Price-Whelan, A. M., Sipőcz, B. M., Günther, H. M., et al. 2018, AJ, 156, 123, doi: 10.3847/1538-3881/aabc4f

Rothman, L. S., Gordon, I. E., Barber, R. J., et al. 2010, JQSRT, 111, 2139, doi: 10.1016/j.jqsrt.2010.05.001

Sánchez-López, A., Alonso-Floriano, F. J., López-Puertas, M., et al. 2019, A\&A, 630, A53, doi: 10.1051/0004-6361/201936084

Shemansky, D. E., Matheson, P., Hall, D. T., Hu, H. Y., \& Tripp, T. M. 1993, Nature, 363, 329, doi: $10.1038 / 363329 \mathrm{a} 0$

Snellen, I. A. G., de Kok, R. J., de Mooij, E. J. W., \& Albrecht, S. 2010, Nature, 465, 1049, doi: $10.1038 /$ nature0911

Stock, J. W., Kitzmann, D., Patzer, A. B. C., \& Sedlmayr, E. 2018, MNRAS, 479, 865, doi: 10.1093/mnras/sty1531

Tamura, M., Suto, H., Nishikawa, J., et al. 2012, in Society of Photo-Optical Instrumentation Engineers (SPIE) Conference Series, Vol. 8446, Ground-based and Airborne Instrumentation for Astronomy IV, ed. I. S. McLean, S. K. Ramsay, \& H. Takami, 84461T, doi: $10.1117 / 12.925885$

Tamuz, O., Mazeh, T., \& Zucker, S. 2005, MNRAS, 356, 1466, doi: 10.1111/j.1365-2966.2004.08585.x

Todd Clancy, R., Sandor, B. J., García-Muñoz, A., et al. 2013, Icarus, 226, 272, doi: 10.1016/j.icarus.2013.05.035

Virtanen, P., Gommers, R., Oliphant, T. E., et al. 2020, Nature Methods, 17, 261, doi: https://doi.org/10.1038/s41592-019-0686-2

Visscher, C., Lodders, K., \& Fegley, Bruce, J. 2006, ApJ, 648, 1181, doi: 10.1086/506245

von Braun, K., Boyajian, T. S., Kane, S. R., et al. 2012, ApJ, 753, 171, doi: 10.1088/0004-637X/753/2/171 von Essen, C., Mallonn, M., Welbanks, L., et al. 2019, A\&A, 622, A71, doi: 10.1051/0004-6361/201833837 
Wang, J., Mawet, D., Fortney, J. J., et al. 2018, AJ, 156, 272, doi: 10.3847/1538-3881/aae47b

Webb, R. K., Brogi, M., Gandhi, S., et al. 2020, MNRAS, 494, 108, doi: 10.1093/mnras/staa715

Yan, F., Casasayas-Barris, N., Molaverdikhani, K., et al. 2019, A\&A, 632, A69, doi: 10.1051/0004-6361/201936396
Yan, F., Pallé, E., Reiners, A., et al. 2020, A\&A, 640, L5, doi: 10.1051/0004-6361/202038294

Yan, F., Wyttenbach, A., Casasayas-Barris, N., et al. 2021, A\&A, 645, A22, doi: 10.1051/0004-6361/202039302

Yousefi, M., Bernath, P. F., Hodges, J., \& Masseron, T. 2018, JQSRT, 217, 416, doi: 10.1016/j.jqsrt.2018.06.016 